\title{
SnowWatch: a multi-modal citizen science application
}

\author{
Roman Fedorov, Piero Fraternali, and Chiara Pasini \\ Politecnico di Milano, \\ Dipartimento di Elettronica, Infomazione e Bioingegneria \\ Piazza Leonardo da Vinci, 32, Milan, Italy \\ ffirst.last\}@polimi.it
}

\begin{abstract}
The demo presents SnowWatch, a citizen science system that supports the acquisition and processing of mountain images for the purpose of extracting snow information, predicting the amount of water available in the dry season, and supporting a multi-objective lake regulation problem. We discuss how the proposed architecture has been rapidly prototyped using a general-purpose architecture to collect sensor and user-generated Web content from heterogeneous sources, process it for knowledge extraction, relying on the contribution of voluntary crowds, engaged and retained with gamification techniques.
\end{abstract}

Keywords: Rapid prototyping, citizen science, crowdsourcing

\section{Introduction}

The diffusion of mobile devices, social networks and online games has spawned a novel generation of hybrid applications, associated under the generic label of "citizen science", which harness the online, voluntary contribution and cooperation of common people for the resolution of complex tasks in a variety of domains, including computer vision, transport, environment monitoring, bio-medical research, and more [4]. The common traits of these applications include: (i) the use of people as soft sensors to acquire data about the physical environment to be monitored or analysed (ii) the fusion of heterogeneous data, coming not only from people, but also from conventional sensors (iii) the need of validating data, for improving input accuracy and training/tuning data processing algorithms (iv) the provision of mechanisms for recruiting, engaging, and retaining people, who contribute voluntarily and should be acknowledged for their participation.

This demo presents SnowWatch, a citizen science application for the acquisition and processing of mountain images for the purpose of extracting snow

The work has been partially funded by the EC and Regione Lombardia, under the FP7 SmartH2O and FESR PROACTIVE projects. 
information, predicting the amount of water available in the dry season, and supporting a multi-objective lake regulation problem.

SnowWatch has been realized on top of a Web/mobile software architecture for the rapid development of citizen science applications, based on three main tiers: (i) a back-end that supports the composition of data processing workflows, by the collation of independent, loosely-coupled data acquisition and analysis modules (ii) a client tier, which can hosts multiple applications, fixed Web and mobile, that implement common interfaces for publishing tasks to workers and collecting their contributions. (iii) a middle tier independent of both the data processing back-end and of the client crowdsourcing applications, which factors out the engagement policies and achievement rewarding rules enacted to secure people participation and durable commitment.

\section{The SnowWatch application and rapid prototyping architecture}

The SnowWatch project [1], in which the architecture and application have been developed, supports the low cost analysis of environmental mountain phenomena. Several state-of-the-art methods try to virtualize permanent measurement stations, e.g, for snow and other mountain environment parameters, through aerial and terrestrial image analysis [3], but require high quality images, which are scarce and costly, and are insufficient to build and calibrate a really usable mountain environmental model. On the other hand, the visual UGC publicly available on the Web is almost unlimited and a significant portion of it consists of outdoor photos. Based on such content, SnowWatch tackles the problem of mountain environment monitoring with a Citizen Science application for the collection of public mountain images and the extraction of snow indexes usable in water prediction models. The system crawls a large number of images from content sharing sites and touristic webcams, classifies those images that portrait mountain peaks and contain the location of shooting, identifies visible peaks by automatically aligning each image to a synthetic rendition computed from a public digital terrain model, finds the pixels of each peak that represent snow and calculates useful snow indexes (e.g, minimum snow altitude). These indexes are then used to feed existing water prediction models and compared with other official sources of information. Crowdsourcing is also employed, for three tasks: validating the classification of images that contain visible mountain profiles; validating the peak identification computed automatically; collecting images on demand, e.g., portraying mountain for which there is not enough UGC and no webcams are available.

SnowWatch is implemented by instantiating the general architecture of Figure 1 , reusing standard components and adding domain-specific services. The image processing and computer vision algorithms used in the project are explained in detail in $[1,2]$.

Two Image source connectors and one Data Aggregator have been configured, for acquiring images from sharing sites, webcams, and users. A Flickr 

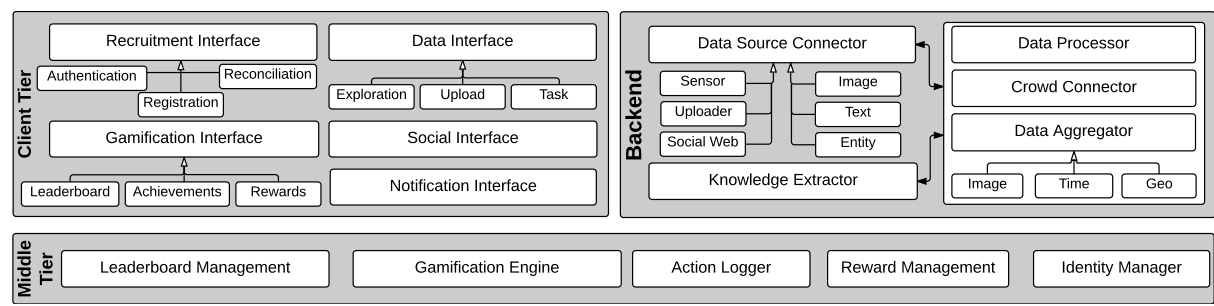

Fig. 1. Citizen science development architecture used in SnowWatch

Crawler specializes the generic, keyword-search, image source connector with three filtering criteria: the photo must be geo-tagged and located within a rectangular region provided in input, and the altitude of the shooting location must be higher than a minimum threshold. The Mountain webcam crawler specializes the generic webcam source connector with a filtering step. Since cloudy meteorological conditions are very common at high altitudes, the connector discards images with bad weather conditions. The Image aggregator specializes the Data Aggregator interface to collapse a set of input mountain images, taken in good weather conditions, into one daily median image. This steps removes even small transient clouds and boosts the precision of the subsequent image processing steps that must identify accurately the edges of a mountain range.

The Data Processor interface is the one most heavily specialized, to incorporate the domain-specific algorithms for mountain image analysis. The realized specializations include: 1. Mountain photo classifier, used in the Mountain relevance classification automatic task, applies to each candidate photo a supervised learning Support Vector Machine (SVM) binary classification step, discarding the photos that do not contain a clearly visible mountain profile. 2. Photo orientation estimator, used in the Mountain peak identification automatic task, given as input a geo-tagged photo, estimates the direction of the camera using a matching algorithm on the photo edge maps and a rendered view of the mountain silhouettes that should be seen from the photographers point of view [?]. 3. Snow pixel detector, used in the Snow mask computation and automatic task, receives as input an image for which the direction of shooting is known and computes for each pixel representing the terrain a Boolean label (snow, no-snow). It outputs a binary snow mask representing for each pixel the presence or absence of snow. The Crowd Connector has been instantiated as follows: a task GUI has been added in the client tier, enabling the execution of the task. And a crowd connector has been allocated in the back-end, to support the recruitment of the contributors.

Finally, the Knowledge Extractor has been instantiated with a Snow Index Computation service. This service transforms the snow masks into real-valued indexes that represent virtual snow measurements (e.g. area covered by snow in $\mathrm{km}^{2}$ or minimum altitude of snow in $\mathrm{m}$ ). The time series of these indexes produced for the mountains of interest are the final output of SnowWatch and can 
be fed to environmental machine learning models that exploit this information for predicting water availability and taking decisions in water allocation problems.

The Middle Tier (Gamification Engine) has been used to configure the Gamification Data Model, by creating: i) actions that can be performed by a user (e.g. upload a photo, share a photo with friends, comment a photo, validate a photo, manually align a photo, etc); ii) achievements and actions required to obtain them; iii) rewards that can be provided to users for their achievements.

The Client Tier of the application has been customized by adding the GUIs needed for supporting the execution of human tasks and an exploratory Web portal interface for the general public:

- Exploratory Web portal ${ }^{1}$ : it customizes the Exploration Data Interface to support browsing the geo-located image collection, in two ways: with a map view, placing the images on a map in the positions they were shot; and with a gallery view that publishes all images into a scrollable grid.

- Mountain photo classification validation: the user can label as negative (does not contain mountains) a photo that was erroneously classified as positive.

- Manual photo-to-terrain-model alignment: the user can adjust the automatically computed alignment of the photo to the rendered terrain view.

- Mountain photo shooter: it is a mobile application supporting the human task whereby the user can take photos of mountains with the peak names automatically overlaid onto the image.

At the moment, SnowWatch has collected more than $174 k$ user-generated photos and $30 M$ webcam images. Over 3500 webcams in the Alpine region have been identified and $\sim 2000$ of them have been registered to the application as they point to mountains of interest. The utility of the SnowWatch virtual snow indexes has been validated in a simulation exercise about the regulator of the water level of the Como lake, optimizing a two-objectives problem: flood risk vs water availability. The simulation shows that using the information of the virtual snow indexes helps the regulation of the lake to make more informed decisions on water release and thus attain a policy closer to the optimum Pareto frontier.

\section{References}

1. Fedorov, R., Camerada, A., Fraternali, P., Tagliasacchi, M.: Estimating snow cover from publicly available images. IEEE Transactions on Multimedia PP(99) (2016)

2. Fedorov, R., Fraternali, P., Tagliasacchi, M.: Snow phenomena modeling through online public media. In: Image Processing (ICIP), 2014 IEEE International Conference on. pp. 2174-2176. IEEE (2014)

3. Garvelmann, J., Pohl, S., Weiler, M.: From observation to the quantification of snow processes with a time-lapse camera network. Hydrol. Earth Syst. Sci 17(4), 1415-1429 (2013)

4. Memarsadeghi, N.: Citizen science [guest editors' introduction]. Computing in Science Engineering 17(4), 8-10 (July 2015)

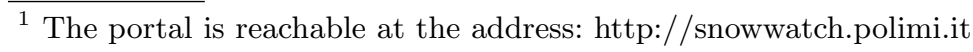

